1. Brown. W.L. Astrophys. J. 207. L.209 (1976).

2. Durrance, S.T.\& Moos. H.W. Nature 299. 426 ( 1982 )

4. Reynolds. R.T. \& Summers, A.L. J. geophys. Res. 70. 199 (1965).

5. Hubbard, W.B. \& MacFarlane, J.J. J. geophys. Res. 85 225 (1980)

6. Podolak, M. \& Reynolds, R.T. Icarus 46, 40 (1981)

7. Stevenson. D.J. A. Rev. Earth planet. Sci. 10. 257 (1982)

8. Midgley, J.E. \& Davis. L. Jr J. geophys. Res. 67, 499 (1962)

10. Hill. T.E. \& Dessler. A.J. Science 227, 1466 (1985)

11. Curtis, S.A. Nature 318, 47 (1985)

11. Curtis. S.A. Nanre 318. $47(1$

Kaiser, M.L. \& Desch, M.D. Rev. Geophys. Space Phys.
22. 373 (1984).

\section{Solar wind interaction with Uranus' atmosphere} radio emission from Uranus even from within 1 astronomical unit may be explained along the lines suggested by $\mathrm{Ax}$ ford and Vasyliunas (preceding letter) by supposing that the surface magnetic field is approximately 0.01 to 0.1 gauss, but it is also possible that Uranus simply has no intrinsic magnetic field. In that case, the solar wind may impinge directly on the neutral upper atmosphere, as it does on Venus. I shall explore briefly the consequences of this assumption for the forthcoming encounter between Voyager 2 and Uranus.

Figure 1 shows the circumstances likely to be encountered by Voyager 2. The scavenging effect of the solar wind should produce a wake of exospheric ions $\left(\mathrm{H}_{2}^{+}\right.$, $\mathrm{He}, \mathrm{H}_{3}{ }^{+}, \mathrm{CH}_{4}^{+}$and so on) converging behind the planet ${ }^{1.2}$. As well as this trail of atmospheric ions, Voyager 2 must be expected to measure a flux of heavy ions sputtered from the particles of which the rings are made. There is thus a chance that some information on the surface composition of these narrow rings may be obtained, while the total area of the optically opaque ring system (approximately 4 $\times 10^{17} \mathrm{~cm}^{2}$, or more than three times the projected area of Uranus itself) may con-
3. Clarke. J.T. Astrophys. J. 263. L105 (1982)

9. Siscoe. G. L. Icarus 24. 311 (1975)

Sir-The failure of Voyager 2 to detect

stitute a detectable obstacle to the flow of the solar wind.

Even if Uranus itself has no magnetic field, it is to be expected that there will be a certain degree of amplification of the interplanetary field in the neighbourhood of the planet, as there is in the neighbourhood of Venus; we estimate e $^{3 .+}$ that a peak field of 4 nanotesla in the sub-solar regions may be induced in this way. Moreover, the interplanetary field lines will be expected to be draped around Uranus, forming a downstream magnetotail, as at Venus and as observed by Voyager 1 during its close encounter with the Saturn satellite Titan.

The intense ultraviolet emission from Uranus must be explained within the same framework. The total energy flux of the solar wind at the projected cross section of Uranus is of the order of $5 \times 10^{4} \mathrm{~W}$, which is several orders of magnitude less than the flux required if the observed Lyman- $\alpha$ emission is to be excited directly by charged particle impact. This is the argument by which Hill and Dessler concluded that a mechanism based on auroral excitation would require a sizeable magnetosphere with a surface magnetic field of a few gauss, but if the function of the charged particle impact is to fragment $\mathrm{H}_{2}$ molecules, which then give rise to ultraviolet emission by the resonant scattering of solar radiation, the energy requirements are less critical. Even so, the upper limit of $5 \times 10^{4} \mathrm{~W}$ in the case of a Venuslike interaction is a stringent condition on the energy deliverable to the atmosphere of Uranus by the solar wind.

Support for the view that the Lyman- $\alpha$ flux may arise from processes other than the direct impact of magnetospheric electrons has been provided by the earlier encounters of Voyager spacecraft with Titan and by investigations of the surroundings of Venus, chiefly by means of Soviet spacecraft. One idea is that the effect of the enhanced curvature of the magnetic

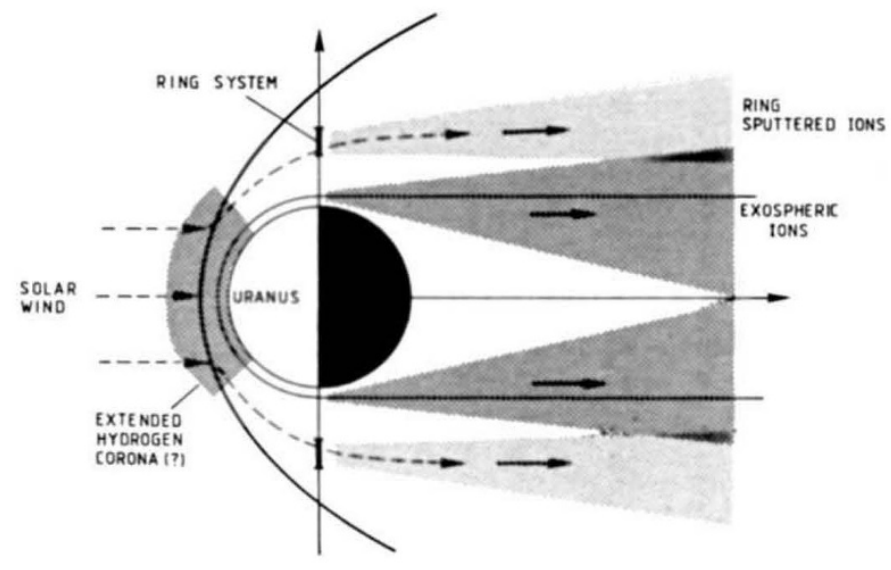

Fig. 1 A schematic view of possible direct solar wind interaction with the neutral atmosphere of Uranus. Points of interest are the upstream ionization interaction with the (putative) extended corona of $\mathrm{H}$ atoms; the plasma mantle formed by pickup of the exospheric ions; and the cylindrical sheath of sputtered ions from the rings. field lines around Titan is to increase the flux of suprathermal magnetospheric electrons on the atmosphere of the satellite, which is apparent both in the increased flux of ultraviolet radiation from $\mathrm{N}_{2}$ molecules and in the depletion of the flux of magnetospheric electrons above $700 \mathrm{eV}$.

It is important that atmospheric precipitation of charged particles may not be a sufficient explanation. $\mathrm{N}_{2}$ emission has been observed on the dayside disc of Titan and attributed to the effect of solar radiation on the interaction between the magnetospheric plasma and the atmosphere ${ }^{+.6}$. Another possibility is that the circumstances around Titan may resemble those around Venus, and also Uranus, in allowing the enhanced ionization of atmospheric constituents (see refs 7-11).

W.-H. IP

Max-Planck-Institute für Aeronomie, D-3411 Katlenburg-Lindau, FRG

1. Ip. W.-H. in Future Missions in Solar. Heliospheric and Space Plasma Physics (Proc. ESA workshop GarmischPartenkirch) 65 (ESA SP-235, 1985).

2. Gringauz. K.I. in Venus (eds Hunten. D.M. et al.) 779 (University of A rizona Press. Tucson. 1983)

3. Smith, G.R. et al. J. geophys. Res. 87, 1351 (1982)

4. Neubauer, F.M. et al. in Saturn (eds Gehrels. T. \& Matthews, M.S.) 760 (University of Arizona Press. Tucson, 1984).

5. Russell, C.T. \& Vaisberg, O.L. in Venus, (eds Hunten.

D.M. et al.) 873 (University of Arizona Press, Tucson. 1983).

6. Hartle, R.E. Adv. Space Res. 5, 321 (1984).

7. Alfvén. H. Rev. mod. Phys. 37, 652 (1985)

8. Danielsson. L. Phys. Fluids 13, 2288 (1970).

9. Raadu, M.A. Astrophys. Space Sci. 55, 125 (1978).

0. Formisano, V., Galeev. A.A. \& Sagdeev, R.Z. Planet. Space Sci. 30, 491 (1982).

11. Vaisberg, O.L. \& Zeleny, L.M. Icarus 58, 412 (1984).

\section{Archaebacterial terminator}

SIR-In his News and Views article on "The uniqueness of archaebacteria" ( $\mathrm{Na}$ ture 318, 234; 1985) Roger A. Garrett quotes me as having suggested "that five consecutive thymidines might be sufficient for ending the methyl-CoMreductase gene of Methanococcus voltae". That is, in fact, not true. Our findings on the terminator structure in Methanococcus have since been published (Nucleic Acids Res. 13, 6439-6445; 1985). The terminator strongly resembles a eubacterial terminator.

Philipps Universität Marburg,

Albrecht KLEIN Fachbereich Biologie,

Molekulargenetik,

Karl-von-Frisch-Strasse, 3550 Marburg, FRG

R.A. GARRETT REPLIES-This archaebacterial terminator was selected from several reported that appeared noneubacterial in character. Other putative terminators, including those for the ribosomal RNA genes of the extreme halophile Halococcus morrhuae and the thermoproteale Desulfurococcus mobilis, remain exceptional. 